\title{
ON INCREASING-FAILURE-RATE RANDOM VARIABLES
}

\author{
SHELDON M. ROSS, ${ }^{*}$ University of Southern California \\ J. GEORGE SHANTHIKUMAR ***** AND \\ ZEGANG ZHU ****** University of California, Berkeley
}

\begin{abstract}
We provide sufficient conditions for the following types of random variable to have the increasing-failure-rate (IFR) property: sums of a random number of random variables; the time at which a Markov chain crosses a random threshold; the time until a random number of events have occurred in an inhomogeneous Poisson process; and the number of events of a renewal process, and of a general counting process, that have occurred by a randomly distributed time.
\end{abstract}

Keywords: Stochastic ordering; IFR property; DRHR property; renewal process; Markov chain

2000 Mathematics Subject Classification: Primary 62E10

Secondary 60E15

\section{Introduction and summary}

The increasing-failure-rate (IFR) property is a well-known and useful concept in reliability theory, dynamic programming, and other areas of applied probability and statistics (see Shaked and Shanthikumar (1994) and Barlow and Proschan (1975)). In much of the literature, it appears as a condition that enables one to prove inequalities, to show structural results of optimal policies in models of manufacturing systems, and so on.

In this paper, we consider a variety of models in applied probability and focus on finding conditions under which certain random variables of interest have the IFR property (or 'are IFR').

In Section 2, we define the notation used throughout the paper, give definitions of relevant stochastic orderings, and list some useful results related to these concepts, which can be found in Shaked and Shanthikumar (1994). Kijima (1989) considered a Markov chain having the property that the larger is the current state then the larger, in the sense of reversed hazard rate order, will be the next state. Starting from a minimum state of such a chain, Kijima showed that the time at which the chain first crosses a fixed state is IFR (see also Shaked and Shanthikumar (1988) and Li and Shaked (1997)). This result implies that the number of events of a renewal process before a fixed time is IFR whenever the underlying interarrival time has a decreasing reversed hazard rate (DRHR) distribution. In Section 3, we prove a general theorem which

\footnotetext{
Received 8 December 2004; revision received 16 February 2005.

* Postal address: Daniel J. Epstein Department of Industrial and Systems Engineering, University of Southern California, 3715 McClintock Avenue, GER 240, Los Angeles, CA 90089-0193, USA. Email address: smross@ usc.edu

** Postal address: Department of Industrial Engineering and Operations Research, 4135 Etcheverry Hall, University of California, Berkeley, CA 94720, USA.

*** Email address: shanthikumar@ieor.berkeley.edu

**** Email address: zhuzgang@ieor.berkeley.edu
} 
implies that a renewal process having a DRHR interarrival distribution has the property that the number of its events occurring before a random IFR-distributed time (independent of the renewal process) is itself IFR.

In Section 4, we give a Markov chain application of this theorem. Specifically, we show that if the next state of the Markov chain, as a stochastic function of the current state, is increasing in the sense of reversed hazard rate order, and if the increment from the current state to the next state is increasing in the sense of stochastic order, then, starting from a minimum state of the chain, the time that the chain first crosses a random IFR-distributed state is itself IFR. The same condition was used in Li and Shaked (1997) to demonstrate a different result.

For a parallel system of identically distributed items, it is well known that the number of item failures before a fixed time is IFR (specifically, it is binomially distributed). In Section 5, we show that the number of failures before a random time is also IFR, provided that the ratio of the hazard rate functions of this random time to the underlying item lifetimes is increasing. We also consider inhomogeneous Poisson processes in Section 5. Whereas it is known that the number of arrivals before a fixed time is IFR (specifically, it is Poisson distributed), we show that the number of arrivals before a random time is IFR provided that the ratio of the hazard rate function of the random time to the intensity function of the inhomogeneous Poisson process is increasing.

In Section 6, we prove IFR results in two models. In the first model, an urn contains target balls, each of which has weight $w$, and nontarget balls, each of which has weight 1 . Balls are randomly withdrawn in a manner such that the probability that any remaining ball is the next to be removed is given by its weight divided by the sum of the weights of all balls that remain. The withdrawals continue until all the target balls have been removed. For this model, we prove that the total number of nontarget balls that are removed from the urn is IFR. The second model is a coalescing model, in which a specified number of balls are put into boxes, with each ball's box being both independently chosen and equally likely to be any of the boxes. All balls falling into the same box are then coalesced (or merged) into a single ball, and the process repeats itself (possibly with fewer balls). We show that the number of balls that remain after $k$ stages is IFR and that the number of repeats until all balls have coalesced into one ball is IFR.

Barlow and Proschan (1975) showed that the sum of a fixed number of independent IFR random variables is IFR, Shanthikumar (1988) showed that the sum of a geometric number of independent, identically distributed decreasing-failure-rate (DFR) random variables is DFR, and Kijima (1992) showed that, for a renewal process with independent, identically distributed and DFR or IFR interarrival times whose distribution is of continuous phase type with two phases, the associated renewal density is respectively decreasing or increasing. In Section 7, we show that the sum of a random IFR-distributed number of heterogeneous exponential random variables is IFR provided that the rates of the exponential random variables are increasing. Similarly, the sum of a random DFR-distributed number of heterogeneous exponential random variables is DFR provided that the rates of the exponential random variables are decreasing. Therefore, an IFR-distributed sum of a sequence of heterogeneous IFR random variables may be strictly DFR (note that geometric and exponential random variables are both IFR and DFR).

For an inhomogeneous Poisson process with intensity function $\lambda(t)$, let $R(t)=\int_{0}^{t} \lambda(s) \mathrm{d} s$. Pellery et al. (2000) showed that if $R(t)$ and $\lambda(t) \mathrm{e}^{-R(t)}$ are both $\log$-concave, then the epoch time of a fixed number of arrivals is a random variable with the increasing likelihood ratio (ILR) property, which is a stronger property than IFR. Also, Kochar (1990) showed that if $\lambda(t)$ is increasing, then the epoch time of a fixed number of arrivals is IFR. We generalize this to show that if $\lambda(t)$ is increasing, then the epoch time of a random IFR-distributed number of 
arrivals is IFR. Similarly, if $\lambda(t)$ is decreasing then the epoch time of a random DFR-distributed number of arrivals is DFR.

\section{Preliminaries}

In this paper, we assume all random variables to be nonnegative, and write $X \stackrel{\mathrm{D}}{=} Y$ to indicate that the random variables $X$ and $Y$ have the same distribution. For any random variable $X$, we define

$$
\begin{aligned}
F_{X}(x) & =\mathrm{P}(X \leq x), & & x \geq 0, \\
\bar{F}_{X}(x) & =1-F_{X}(x), & & x \geq 0, \\
F_{X}^{-1}(u) & =\sup \left\{y: F_{X}(y) \leq u\right\}, & & u \in[0,1] .
\end{aligned}
$$

The following lemma is a known result.

Lemma 2.1. For any random variable $X$, if $U$ is a uniform $[0,1]$ random variable then

$$
F_{X}^{-1}(U) \stackrel{\mathrm{D}}{=} X
$$

If $X$ is a continuous random variable, we use $f_{X}(\cdot)$ to denote its probability density function. If $X$ is a discrete random variable, we use $\left(p_{X}(\cdot)\right)$ to denote its probability mass function. We will use $f(x) \uparrow x$ to mean that the function $f$ is nondecreasing in $x$ from below, and $f(x) \downarrow x$ to mean that $f$ is nonincreasing in $x$ from below.

Definition 2.1. For any continuous random variable $X$, we define its hazard rate function and reversed hazard rate function as

$$
r_{X}(x)=\frac{f_{X}(x)}{\bar{F}(x)} \quad \text { and } \quad q_{X}(x)=\frac{f_{X}(x)}{F_{X}(x)},
$$

respectively. We say that $X$ is IFR if $r_{X}(x) \uparrow x$, DRHR if $q_{X}(x) \downarrow x$, DFR if $r_{X}(x) \downarrow x$, and ILR if $f_{X}(x)$ is log-concave in $x$.

Definition 2.2. For any continuous random variables $X$ and $Y$, we define the following stochastic orderings.

(a) $X \leq_{\text {st }} Y$ if $\bar{F}_{X}(x) \leq \bar{F}_{Y}(x)$ for all $x \in \mathbb{R}^{+}$.

(b) $X \leq \operatorname{lr} Y$ if $f_{Y}(x) / f_{X}(x) \uparrow x$.

(c) $X \leq_{\mathrm{hr}} Y$ if $r_{X}(x) \geq r_{Y}(x)$ for all $x \in \mathbb{R}^{+}$.

(d) $X \leq_{\text {rh }} Y$ if $q_{X}(x) \leq q_{Y}(x)$ for all $x \in \mathbb{R}^{+}$.

If we use the variable $i$ with domain $\mathbb{Z}^{+}$to replace $x$ with domain $\mathbb{R}^{+}$, and use $p_{X}(\cdot)$ to replace $f_{X}(\cdot)$, we can define the same rate concepts and stochastic orderings for discrete random variables.

Suppose that $\left\{X_{r}\right\}_{r \in \delta}$ is a set of random variables, where $\&$ is an ordered set. In the following, we will write $X_{r} \uparrow r$ to indicate that $X_{r_{1}} \leq X_{r_{2}}$ almost surely for all $r_{1}<r_{2}$, and write $X_{r} \uparrow_{\text {st }} r$ to indicate that $X_{r_{1}} \leq_{\mathrm{st}} X_{r_{2}}$ for all $r_{1}<r_{2}$ (i.e. $X_{r}$ is nondecreasing in $r$ in the 'st' ordering sense). Other ordering notation is similarly defined. We use $[X \mid A]$ to denote the random variable $X$ conditioned on $A$. 
Chapter 1 of Shaked and Shanthikumar (1994) contains proofs of the following four lemmas.

Lemma 2.2. For a random variable $X$, that $X$ is ILR implies that $X$ is both IFR and DRHR, and $X$ is IFR if and only if $[X-x \mid X>x] \downarrow_{\text {st }} x$.

It is easy to check that geometric and exponential random variables are ILR and, thus, that they are both IFR and DRHR.

Lemma 2.3. For random variables $X$ and $Y$, if $X \leq_{\mathrm{lr}} Y$ then $X \leq_{\mathrm{hr}} Y$ and $X \leq_{\mathrm{rh}} Y$. Furthermore, if $X \leq_{\mathrm{hr}} Y$ or $X \leq_{\mathrm{rh}} Y$ then $X \leq_{\mathrm{st}} Y$.

Lemma 2.4. For random variables $X$ and $Y$,

$$
\begin{aligned}
X \leq_{\mathrm{st}} Y & \Leftrightarrow F_{X}^{-1}(u) \leq F_{Y}^{-1}(u) \quad \text { for all } u \in[0,1] \\
& \Leftrightarrow \text { there exist an } \bar{X} \stackrel{\mathrm{D}}{=} X \text { and } a \bar{Y} \stackrel{\mathrm{D}}{=} Y \text { such that } \bar{X} \leq \bar{Y} \text { almost surely } \\
& \Leftrightarrow \mathrm{E} f(X) \leq \mathrm{E} f(Y) \quad \text { for all } f(x) \uparrow x .
\end{aligned}
$$

Lemma 2.5. For any random variable $X$, we have $[X \mid X \leq x]$ ss $x$, and for random variables $X$ and $Y$, if $X \leq_{\mathrm{rh}} Y$ then $[X \mid X \leq x] \leq_{\mathrm{rh}}[Y \mid Y \leq x]$ for all $x$.

\section{IFR property of counting at random times}

Lemma 3.1. Suppose that $V, W$, and $T$ are continuous random variables such that $T$ is independent of $(V, W)$ and $W \geq V$ almost surely. Also suppose that $Z:=[V \mid T \geq V]$ and that $U_{1}$ and $U_{2}$ are uniform $[0,1]$ random variables. Define $W^{x}=F_{[W-x \mid V=x]}^{-1}\left(U_{1}\right)$ and $T^{x}=F_{[T-x \mid T \geq x]}^{-1}\left(U_{2}\right)$. If $Z, U_{1}$, and $U_{2}$ are independent, then

$$
[(W-V, T-V) \mid T \geq V] \stackrel{\mathrm{D}}{=}\left(W^{Z}, T^{Z}\right) .
$$

Proof. By conditioning on the value of $V$ in $[(W-V, T-V) \mid T \geq V]$, we have

$$
\begin{aligned}
\mathrm{P}(W & -V \leq x, T-V \leq y \mid T \geq V) \\
& =\int_{z} \mathrm{P}(W-z \leq x, T-z \leq y \mid T \geq z, V=z) f_{[V \mid T \geq V]}(z) \mathrm{d} z \\
& =\int_{z} \mathrm{P}(W-z \leq x \mid V=z) \mathrm{P}(T-z \leq y \mid T \geq z) f_{[V \mid T \geq V]}(z) \mathrm{d} z \\
& =\int_{z} \mathrm{P}\left(W^{z} \leq x\right) \mathrm{P}\left(T^{z} \leq y\right) f_{Z}(z) \mathrm{d} z \\
& =\int_{z} \mathrm{P}\left(W^{z} \leq x, T^{z} \leq y\right) f_{Z}(z) \mathrm{d} z \\
& =\mathrm{P}\left(W^{Z} \leq x, T^{Z} \leq y\right)
\end{aligned}
$$

from which the result follows.

Lemma 3.2. If $X \leq_{\mathrm{rh}} Y$ and $T$ is independent of $(X, Y)$, then $[T \mid T \geq X] \leq_{\operatorname{lr}}[T \mid T \geq Y]$ and, thus, $[T \mid T \geq X] \leq_{\text {st }}[T \mid T \geq Y]$.

Proof. The result follows directly from the densities of $[T \mid T \geq X]$ and $[T \mid T \geq Y]$ and the fact that, since $X \leq_{\text {rh }} Y$, we have $F_{Y}(x) / F_{X}(x) \uparrow x$ (see Theorem 1.B.14 of Shaked and Shanthikumar (1994)). 
Lemma 3.3. If $X \leq_{\mathrm{rh}} Y$ and $T$ is independent of $(X, Y)$, then $[X \mid T \geq X] \leq_{\mathrm{st}}[Y \mid T \geq Y]$.

Proof. Define $T_{1}=[T \mid X \leq T]$ and $T_{2}=[T \mid Y \leq T]$. From Lemma 3.2, we obtain $T_{1} \leq_{\text {st }} T_{2}$ and, thus, we can assume that $T_{1} \leq T_{2}$ almost surely (from Lemma 2.4). Let $U$ be a uniform $[0,1]$ random variable independent of $T_{1}$ and $T_{2}$, and define $X^{x}=F_{[X \mid X<x]}^{-1}(U)$ and $Y^{x}=F_{[Y \mid Y \leq x]}^{-1}(U)$. Thus, $X^{x} \leq Y^{x}$ for all $x$ (by Lemmas 2.5 and 2.4) and $Y^{x} \uparrow x$ (by Lemmas 2.5 and 2.1), meaning that

$$
X^{T_{1}} \leq Y^{T_{1}} \leq Y^{T_{2}} .
$$

Using the method of Lemma 3.1, we can prove that $[X \mid X \leq T] \stackrel{\mathrm{D}}{=} X^{T_{1}}$ and $[Y \mid Y \leq T] \stackrel{\mathrm{D}}{=}$ $Y^{T_{2}}$. Then, since $X^{T_{1}} \leq Y^{T_{2}}$, we have $[X \mid X \leq T] \leq_{\text {st }}[Y \mid Y \leq T]$ (by Lemma 2.4).

Consider a random sequence $\left\{S_{i}\right\}_{i \geq 1}$ with $S_{i} \uparrow i$, and define $N(t)=\sup \left\{i: S_{i} \leq t\right\}$. The following lemma shows that, for a sequence of independent and IFR or DRHR interarrival random variables, the number of arrivals up to a fixed time is nondecreasing in this fixed time in the reversed hazard ratio ordering sense or the hazard ratio ordering sense, respectively.

Lemma 3.4. Let $\left\{X_{i}\right\}_{i \geq 1}$ be a sequence of independent IFR or DRHR random variables, let $S_{0}=0$, and let $S_{n}=\sum_{i=1}^{n} X_{i}, n \geq 1$. Then $N(t) \uparrow_{\mathrm{rh}} t$ or $N(t) \uparrow_{\mathrm{hr}} t$, respectively.

Proof. Using the facts that $S_{n} \leq_{\mathrm{hr}} S_{n+1}$ (see Theorem 1.B.7 of Shaked and Shanthikumar (1994)) and $\{N(t)<n\}=\left\{S_{n}>t\right\}$, we have

$$
\frac{\mathrm{P}\left(N\left(t_{1}\right)<n\right)}{\mathrm{P}\left(N\left(t_{2}\right)<n\right)}=\frac{\mathrm{P}\left(S_{n}>t_{1}\right)}{\mathrm{P}\left(S_{n}>t_{2}\right)} \leq \frac{\mathrm{P}\left(S_{n+1}>t_{1}\right)}{\mathrm{P}\left(S_{n+1}>t_{2}\right)}=\frac{\mathrm{P}\left(N\left(t_{1}\right)<n+1\right)}{\mathrm{P}\left(N\left(t_{2}\right)<n+1\right)}
$$

for $t_{2}<t_{1}$. From Theorem 1.B.14 of Shaked and Shanthikumar (1994), it follows that $N\left(t_{2}\right) \leq_{\mathrm{rh}}$ $N\left(t_{1}\right)$. The DRHR result can be proved similarly.

We now prove an important theorem that is used extensively throughout the paper.

Theorem 3.1. The random variable $N(T)$ is IFR if the following conditions are satisfied:

(a) $T$ is IFR and independent of $\left\{S_{i}\right\}_{i \geq 1}$;

(b) $S_{i} \uparrow_{\mathrm{rh}} i$;

(c) $\left[S_{i+1}-S_{i} \mid S_{i}=x\right] \leq_{\text {st }}\left[S_{j+1}-S_{j} \mid S_{j}=y\right]$ for all $j>i$ and $y \geq x \geq 0$.

Proof. Define $Z_{i}=\left[S_{i} \mid S_{i} \leq T\right], i \geq 1$. Then $Z_{i} \uparrow$ st $i$ and, so (by condition (b) and Lemma 3.3), we can assume that $Z_{i} \uparrow i$ (by Lemma 2.4). Let $U_{1}$ and $U_{2}$ be uniform[0,1] random variables independent of one another and $\left\{Z_{i}\right\}_{i \geq 1}$, and define

$$
T^{x}=F_{[T-x \mid T \geq x]}^{-1}\left(U_{1}\right) \quad \text { and } \quad S_{i+1}^{x}=F_{\left[S_{i+1}-x \mid S_{i}=x\right]}^{-1}\left(U_{2}\right)
$$

for all $i \geq 1$. Then $T^{Z_{i}} \downarrow i$ (by condition (a) and Lemma 2.2) and $S_{i+1}^{Z_{i}} \leq S_{j+1}^{Z_{j}}$ for all $i<j$ (by condition (c) and the fact that $Z_{i} \uparrow i$ ), and, thus,

$$
\left\{S_{i+1}^{Z_{i}} \geq T^{Z_{i}}\right\} \subseteq\left\{S_{j+1}^{Z_{j}} \geq T^{Z_{j}}\right\}
$$

However, by Lemma 3.1 we have

$$
\begin{gathered}
{\left[\left(S_{i+1}-S_{i}, T-S_{i}\right) \mid T \geq S_{i}\right] \stackrel{\mathrm{D}}{=}\left(S_{i+1}^{Z_{i}}, T^{Z_{i}}\right),} \\
{\left[\left(S_{j+1}-S_{j}, T-S_{j}\right) \mid T \geq S_{j}\right] \stackrel{\mathrm{D}}{=}\left(S_{j+1}^{Z_{j}}, T^{Z_{j}}\right),}
\end{gathered}
$$


so, for all $i<j$,

$$
\begin{aligned}
r_{N(T)}(i) & :=\mathrm{P}(N(T)=i \mid N(T) \geq i) \\
& =\mathrm{P}\left(S_{i+1}>T \mid S_{i} \leq T\right) \\
& =\mathrm{P}\left(S_{i+1}-S_{i}>T-S_{i} \mid S_{i} \leq T\right) \\
& =\mathrm{P}\left(S_{i+1}^{Z_{i}} \geq T^{Z_{i}}\right) \\
& \leq \mathrm{P}\left(S_{j+1}^{Z_{j}} \geq T^{Z_{j}}\right) \\
& =\mathrm{P}\left(S_{j+1}>T \mid S_{j} \leq T\right) \\
& =r_{N(T)}(j) .
\end{aligned}
$$

It follows that $N(T)$ is IFR.

The following corollary shows that if all elements of a sequence of stochastically nondecreasing, independent interarrival random variables have decreasing reversed hazard rates, then the number of arrivals before an IFR random time is itself IFR.

Corollary 3.1. If $X_{i} \uparrow_{\mathrm{st}} i$ and $X_{i}$ is DRHR for all $i, T$ is IFR. If $\left\{X_{i}\right\}, i \geq 1$, and $T$ are all independent, then $\sup \left\{i: \sum_{k=1}^{i} X_{k} \leq T\right\}$ is IFR.

Proof. Since the $X_{i}$ are all DRHR, we have $\sum_{k=1}^{i} X_{k} \uparrow_{\mathrm{rh}} i$ (see Theorem 1.B.25 of Shaked and Shanthikumar (1994)). Thus, the conditions of Theorem 3.1 are all satisfied, proving the result.

This corollary shows that, for a renewal process with DRHR interarrival distribution, the number of arrivals till a random time is itself IFR if this random time is an IFR random variable.

All above results remain true when the $S_{i}$, the $X_{i}$, and $T$ are discrete: the proofs follow the same steps.

\section{Application to the first passage time of Markov chains}

Lemma 4.1. Suppose that $\Theta_{1}, \Theta_{2}$, and $X(\theta), \theta \geq 0$, are all nonnegative random variables. If $\Theta_{1} \leq_{\mathrm{rh}} \Theta_{2}$ and $X(\theta) \uparrow_{\mathrm{rh}} \theta$, then $X\left(\Theta_{1}\right) \leq_{\mathrm{rh}} X\left(\Theta_{2}\right)$.

The proof follows from that of Lemma 2.1 of Shanthikumar (1988) by letting $t_{i j}=\mathbf{1}_{\{j \geq i\}}$ and $t_{i j}^{-1}=\mathbf{1}_{\{i=j\}}-\mathbf{1}_{\{j=i+1\}}$ (in the latter proof), where $\mathbf{1}_{A}$ is the indicator of the event $A$.

Let $\left\{X_{n}^{i}\right\}_{n \geq 0}$ be a Markov chain with state space $\{1, \ldots, M\}$ ( $M$ could be infinite) and transition matrix $\boldsymbol{P}$, and start from state $i$, i.e. $X_{0}^{i}=i$.

Lemma 4.2. If $X_{1}^{i} \uparrow_{\mathrm{rh}} i$ then

(a) $\Theta_{1} \leq_{\mathrm{rh}} \Theta_{2}$ implies that $X_{n}^{\Theta_{1}} \leq_{\mathrm{rh}} X_{n}^{\Theta_{2}}$ for all $n \geq 0$, and

(b) $X_{n}^{1} \uparrow$ rh $n$.

Proof. (a) The result is trivial for $n=0$. Suppose the result is true for $n=k$, and define $Y(i)=X_{1}^{i}$. Then, by the Markov property, we have $X_{k+1}^{\Theta_{j}} \stackrel{\text { D }}{=} Y\left(X_{k}^{\Theta_{j}}\right), j=1,2$. Thus, $X_{k}^{\theta_{1}} \leq_{\mathrm{rh}} X_{k}^{\Theta_{2}}$ by induction, and we obtain $X_{k+1}^{\Theta_{2}} \leq_{\mathrm{rh}} X_{k+1}^{\Theta_{1}}$ from Lemma 4.1.

(b) Clearly, $X_{0}^{1}=1 \leq_{\mathrm{rh}} X_{1}^{1}$, so, by the result we have just proved and the Markov property, we have $X_{n}^{1} \leq_{\mathrm{rh}} X_{n}^{X_{1}^{1}} \stackrel{\mathrm{D}}{=} X_{n+1}^{1}$. 
Define $T=\inf \left\{n: X_{n}^{1}=M\right\}$ to be the time that the Markov chain first reaches the maximum state if it started from the minimum state. Theorem 5.1 of Kijima (1989) shows that, if the one-period end state (i.e. the state at time 1) is nondecreasing in the starting state, in the sense of reversed hazard ratio ordering, then $T$ is an IFR random variable. This is our next theorem, which we prove using a new approach.

Theorem 4.1. If $X_{1}^{i} \uparrow_{\mathrm{rh}} i$ then $T$ is IFR.

Proof. Without loss of generality, we assume $M$ to be the absorbing state, i.e.

$$
\mathrm{P}\left(X_{1}^{M}=M\right)=1,
$$

and write

$$
\bar{X}_{n}^{1}=\left[X_{n}^{1} \mid X_{n}^{1} \leq M-1\right] .
$$

Then, by Lemma 4.2 and Lemma 2.5, we have $\bar{X}_{n}^{1} \uparrow_{\mathrm{rh}} n$ and, thus, $X_{1}^{\bar{X}_{n}^{1}} \uparrow_{\mathrm{st}} n$ by Lemma 4.2. Hence,

$$
\begin{aligned}
\mathrm{P}(T=n \mid T \geq n) & =\mathrm{P}\left(X_{n}^{1}=M \mid X_{n-1}^{1} \leq M-1\right) \\
& =\mathrm{P}\left(X_{1}^{\bar{X}_{n-1}^{1}}=M\right) \\
& =\mathrm{P}\left(X_{1}^{\bar{X}_{n-1}^{1}} \geq M\right) .
\end{aligned}
$$

Since $\mathrm{P}\left(X_{1}^{\bar{X}_{n-1}^{1}} \geq M\right) \uparrow n$, this shows that $T$ is IFR.

The following corollary shows that the number of periods elapsed until the Markov chain, beginning from the maximum state, first reaches the minimum state is IFR, provided that the one-period end state is increasing in starting state, in the sense of hazard ratio ordering.

Corollary 4.1. For finite $M$, let $\hat{T}=\inf \left\{n: X_{n}^{M}=1\right\}$. If $X_{1}^{i} \uparrow_{\mathrm{hr}} i$ then $\hat{T}$ is IFR.

Proof. Let $\bar{X}_{n}^{i}=M+1-X_{n}^{M+1-i}$ and apply Theorem 4.1 to $\left\{\bar{X}_{n}^{1}\right\}_{n \geq 0}$.

Alternatively, assume that the state space is $\mathbb{Z}_{+}$and define $R=\inf \left\{n: X_{n}^{1}>N\right\}$. The following theorem shows that if the random threshold $N$ is an IFR random variable, the oneperiod state increment of the Markov chain is stochastically increasing in the starting state, and the one-period end state is increasing in the starting state in the sense of reversed hazard ratio ordering, then the number of periods needed to cross $N$ is IFR.

Theorem 4.2. Let $N$ be a random variable, independent of $\left\{X_{n}^{1}\right\}_{n \geq 0}$, satisfying the following conditions:

(a) $N$ is IFR;

(b) $X_{1}^{i}-i \uparrow_{\mathrm{st}} i$;

(c) $X_{1}^{i} \uparrow_{\mathrm{rh}} i$.

Then $R$ is IFR.

Proof. From Lemma 4.2, we have $X_{n}^{1} \uparrow_{\mathrm{rh}} n$. Since 1 is the minimum state of the Markov chain, it follows from condition (b) that

$$
\mathrm{P}\left(X_{1}^{i}-i \geq 0\right) \geq \mathrm{P}\left(X_{1}^{1}-1 \geq 0\right)=1 ;
$$

then $X_{1}^{i} \geq i$ almost surely, which implies that $X_{n}^{1} \uparrow n$. If we take $S_{n}=X_{n}^{1}$ and $T=N$ in Theorem 3.1 then, by the Markov property, we find that $R-1$ and, thus, $R$ are IFR. 
Example 4.1. Suppose that $X_{1}^{i}=i+\operatorname{Poisson}\left(\lambda_{i}\right)$, where $\lambda_{i} \uparrow i$. Then, for any IFR random variable $N$ independent of $\left\{X_{n}^{1}\right\}_{n \geq 0}, R=\inf \left\{n: X_{n}^{1} \geq N\right\}$ is also IFR.

\section{Parallel systems and inhomogeneous Poisson processes}

Consider a system of $n$ items working in parallel. The items' lifetimes, $X_{1}, \ldots, X_{n}$, are independent and identically distributed with distribution $F$. Let $T$ be a nonnegative random variable, independent of the lifetimes, with distribution $G$, and denote by $N(T)$ the number of item failures by time $T$.

Corollary 5.1. If $X_{1}$ is $\operatorname{Exp}(\lambda)$ and $T$ is IFR, then $N(T)$ is IFR.

Proof. Since the interfailure times of independent, exponentially distributed lifetimes are independent and exponentially distributed, with parameter $(n-i+1) \lambda$ for the $i$ th interfailure time, the result follows from Corollary 3.1.

Let $r_{F}(x)$ and $r_{G}(x)$ denote the failure rate functions of $F$ and $G$, respectively. Then, using the above corollary, we can prove a stronger result: the number of item failures before a random time is IFR if the ratio of the failure rate function of this random time to the item lifetime is nondecreasing.

We denote by $(f(x))_{x}^{\prime}$ the derivative of $f(x)$ with respect to $x$ from below.

Theorem 5.1. For arbitrary distributions $F$ and $G$, if $r_{G}(x) / r_{F}(x) \uparrow x$ then $N(T)$ is IFR.

Proof. Suppose that $H$ is the distribution function of an $\operatorname{Exp}(1)$ random variable; then the $H^{-1}\left(F\left(X_{i}\right)\right)$ are also $\operatorname{Exp}(1)$ random variables. The number of items failing before time $T$ is the same as the number of $X_{i}$ for which $H^{-1}\left(F\left(X_{i}\right)\right)$ is less than $H^{-1}(F(T))$, so, by the previous corollary, we need only prove that $H^{-1}(F(T))$ is IFR. However, we note that

$$
\begin{aligned}
\mathrm{P}\left(H^{-1}(F(T))>x\right) & =\mathrm{P}\left(T>F^{-1}(H(x))\right)=\bar{G}\left(F^{-1}(H(x))\right), \\
\left(-\mathrm{P}\left(H^{-1}(F(T))>x\right)\right)_{x}^{\prime} & =g\left(F^{-1}(H(x))\right) \frac{h(x)}{f\left(F^{-1}(H(x))\right)} \\
& =g\left(F^{-1}(H(x))\right) \frac{1-H(x)}{f\left(F^{-1}(H(x))\right)},
\end{aligned}
$$

where, in the final equality, we have used $h(x)=\mathrm{e}^{-x}=1-H(x)$. If we write $F^{-1}(H(x))=$ $y(x)$ then $1-H(x)=1-F(y(x))$ and, thus, the failure rate function of $H^{-1}(F(T))$ is

$$
r_{G}(y(x)) \frac{1-F(y(x))}{f(y(x))}=\frac{r_{G}(y(x))}{r_{F}(y(x))} .
$$

Clearly, $y(x) \uparrow x$ and, thus, from $r_{G}(x) / r_{F}(x) \uparrow x$ it follows that $r_{G}(y(x)) / r_{F}(y(x)) \uparrow x$. This proves that $N(T)$ is IFR.

From Corollary 3.1, we obtain the following result.

Corollary 5.2. If $\{N(t)\}_{t \geq 0}$ is a homogeneous Poisson process and $T$ is an IFR random variable independent of $\{N(t)\}_{t \geq 0}$, then $N(T)$ is IFR.

We now prove a more general result: the number of arrivals in an inhomogeneous Poisson process before a random time is IFR if the ratio of the failure rate function to the arrival intensity function is nondecreasing. 
Theorem 5.2. Suppose that $\{N(t)\}_{t \geq 0}$ is an inhomogeneous Poisson process with intensity function $\lambda(t)$, and that $T$ is a random variable, independent of $\{N(t)\}_{t \geq 0}$, with distribution $G$ and failure rate $r_{G}(t)$. If $r_{G}(t) / \lambda(t) \uparrow t$ then $N(T)$ is IFR.

Proof. Let $m(t)=\int_{0}^{t} \lambda(t) \mathrm{d} t$ and let $\left\{N^{*}(t)\right\}_{t \geq 0}$ be a homogeneous Poisson process with rate 1. Then $\left\{N^{*}(m(t))\right\}_{t \geq 0} \stackrel{\mathrm{D}}{=}\{N(t)\}_{t \geq 0}$ and, thus, $N^{*}(m(T)) \stackrel{\mathrm{D}}{=} N(T)$. To prove that $N(T)$ is IFR, it is therefore enough to prove that $m(T)$ is IFR, by the previous corollary. To this end, we note that

$$
\begin{aligned}
\mathrm{P}(m(T)>x) & =\mathrm{P}\left(T>m^{-1}(x)\right)=\bar{G}\left(m^{-1}(x)\right), \\
(-\mathrm{P}(m(T)>x))_{x}^{\prime} & =\frac{g\left(m^{-1}(x)\right)}{m^{\prime}\left(m^{-1}(x)\right)}=\frac{g\left(m^{-1}(x)\right)}{\lambda\left(m^{-1}(x)\right)}, \\
r_{m(T)}(x) & =\frac{(-\mathrm{P}(m(T)>x))_{x}^{\prime}}{\mathrm{P}(m(T)>x)}=\frac{r_{G}\left(m^{-1}(x)\right)}{\lambda\left(m^{-1}(x)\right)} .
\end{aligned}
$$

Clearly $m^{-1}(x) \uparrow x$, and, thus, $r_{G}\left(m^{-1}(x)\right) / \lambda\left(m^{-1}(x)\right) \uparrow x$ since $r_{G}(t) / \lambda(t) \uparrow t$. This proves that $N(T)$ is IFR.

\section{Ball drawing and ball dropping models}

\subsection{Ball drawing model}

Suppose that an urn contains balls numbered $1, \ldots, n+m$, with ball $i$ having weight $w_{i}$. Balls are sequentially drawn, without replacement, from the urn according to the following scheme: the probability that a ball still in the urn is drawn next is equal to its weight divided by the sum of the weights of all balls still in the urn. Define $A^{\mathrm{c}}=\{1, \ldots, m\}$ and $A=\{m+1, \ldots, m+n\}$. We are interested in the number of balls in $A^{\mathrm{c}}$, say $N$, that have been withdrawn once all the balls in $A$ have been drawn. Below, we show that this random variable is IFR.

Let $N_{1}(t), N_{2}(t), \ldots, N_{m+n}(t)$ be independent homogeneous Poisson processes with rates $w_{1}, w_{2}, \ldots, w_{m+n}$, respectively. Denote by $T_{1}^{i}$ the first event time in process $i$. If we order the $T_{1}^{i}, i=1, \ldots, m+n$, by magnitude, then the ball drawing process is probabilistically equivalent to the index process $i_{k}, k=1, \ldots, m+n$ (i.e. $T_{1}^{i_{k}}$ is the $k$ th smallest of the $T_{1}^{i}$ ). Assume that $T=\max \left\{T_{1}^{i}: i \in\{m+1, \ldots, m+n\}\right\}$, in which case $N \stackrel{\mathrm{D}}{=} \sum_{i=1}^{m} \mathbf{1}_{\left\{T_{1}^{i} \leq T\right\}}$.

Theorem 6.1. If $w_{1}=w_{2}=\cdots=w_{m}$ and $w_{m+1}=\cdots=w_{m+n}$, then $N$ is IFR.

Proof. Since $T$ is the maximum of independent, identically distributed IFR random variables, it is itself IFR (see Example 9.24 of Ross (2000)). Hence, from Corollary 5.1 we find that $N \stackrel{\mathrm{D}}{=} \sum_{i=1}^{m} \mathbf{1}_{\left\{T_{1}^{i} \leq T\right\}}$ is IFR.

In this model, it is easy to calculate the first two moments of $N$ and, thus, using the results of Barlow and Marshall (1964), (1965), to bound the distribution of $N$.

\subsection{Ball dropping model (coalescing model)}

In this model, $m$ balls are dropped into $n$ boxes independently and with equal probability. The balls that fall in the same box coalesce into one, and the remaining balls are then removed and the process repeated until only one ball remains. We are interested in the following random variables:

(a) the number of balls remaining, $N_{k}^{m}$ say, after $k$ repetitions;

(b) the number of repetitions required before one ball remains, i.e. $N=\inf \left\{k: N_{k}^{m}=1\right\}$. 
Li and Shaked (1997) gave many nice results about this model. Here, we show that the above random variables are both IFR.

Theorem 6.2. $N_{k}^{m}, k \geq 1$, and $N$ are IFR random variables.

Proof. At each discrete time, we drop one ball into the boxes with equal probability. Therefore, the time at which a dropped ball first hits a nonempty box is 1 , and the time between the dropped balls hitting the $j$ th and $(j+1)$ th nonempty boxes is geometric $((n-j) / n)$, $j=1, \ldots, n-1$. This is stochastically increasing and DRHR in $j$. Thus, by Corollary 3.1, the number of nonempty boxes at an IFR time is itself IFR, i.e. $N_{1}^{T}$ is IFR for any IFR $T$. Thus, $N_{1}^{m}$ is IFR since the number of dropped balls is deterministic in $m$ (we can actually prove that $N_{1}^{m}$ is ILR) and, by induction, we find that $N_{k}^{m} \stackrel{\mathrm{D}}{=} N_{1}{ }^{N_{k-1}^{m}}$ is IFR.

Since the geometric distribution is DRHR, we obtain $N_{1}^{i} \uparrow \mathrm{hr} i$ (by Lemma 3.4). If we treat $N_{k}^{m}$ as a Markov chain with time $k$, then, by Corollary 4.1, we find that $N$ is IFR.

\section{IFR property of random sums and random epoch times}

We are now ready to prove that the sum of a random IFR-distributed number of heterogeneous exponential random variables is itself IFR, provided that the rates of the exponential random variables are increasing. Similarly, the sum of a random DFR-distributed number of heterogeneous exponential random variables is DFR, provided that the rates of the exponential random variables are decreasing.

Theorem 7.1. Suppose that $X_{i}$ has distribution $\operatorname{Exp}\left(\lambda_{i}\right), i \geq 1$, and that $T$ and $X_{i}, i \geq 1$, are independent of one another.

(a) If $\lambda_{i} \uparrow i$ and $T$ is discrete and IFR, then $\sum_{i=1}^{T} X_{i}$ is IFR.

(b) If $\lambda_{i} \downarrow i$ and $T$ is discrete and DFR, then $\sum_{i=1}^{T} X_{i}$ is DFR. Then

Proof. (a) Let $S_{n}=\sum_{i=1}^{n} X_{i}$ and $N(t)=\max \left\{i: S_{i} \leq t\right\}$, and write $T^{n}=[T-n \mid T \geq n]$.

$$
\sum_{i=n+1}^{n+T^{n}} X_{i} \downarrow_{\mathrm{st}} n
$$

since $T^{n}, X_{n} \downarrow_{\text {st }} n$, meaning that

$$
\mathrm{P}\left(\sum_{i=n+1}^{n+T^{n}} X_{i}>y\right) \downarrow n .
$$

For $x<z$, we have $N(x) \leq_{\text {rh }} N(z)$ by Lemma 3.4, which gives

$$
[N(x) \mid T>N(x)] \leq_{\mathrm{st}}[N(z) \mid T>N(z)] \quad \text { by Lemma 3.3. }
$$

Therefore, by Lemma 2.4, we have

$$
\begin{aligned}
& \sum_{n} \mathrm{P}\left(\sum_{i=n+1}^{n+T^{n}} X_{i}>y\right) \mathrm{P}(N(x)=n \mid T>N(x)) \\
& \quad \geq \sum_{n} \mathrm{P}\left(\sum_{i=n+1}^{n+T^{n}} X_{i}>y\right) \mathrm{P}(N(z)=n \mid T>N(z)) .
\end{aligned}
$$


Hence, for all $x<z$, we have

$$
\begin{aligned}
& \mathrm{P}\left(\sum_{i=1}^{T} X_{i}-x>y \mid \sum_{i=1}^{T} X_{i}>x\right)=\mathrm{P}\left(\sum_{i=1}^{T} X_{i}-x>y \mid N(x)<T\right) \\
& \quad=\sum_{n} \mathrm{P}\left(\sum_{i=n+2}^{T} X_{i}+\left(S_{n+1}-x\right)>y \mid N(x)=n, T>n\right) \mathrm{P}(N(x)=n \mid T>N(x)) \\
& \quad=\sum_{n} \mathrm{P}\left(\sum_{i=n+1}^{n+T^{n}} X_{i}>y\right) \mathrm{P}(N(x)=n \mid T>N(x)) \\
& \quad \geq \sum_{n} \mathrm{P}\left(\sum_{i=n+1}^{n+T^{n}} X_{i}>y\right) \mathrm{P}(N(z)=n \mid T>N(z)) \\
& \quad=\mathrm{P}\left(\sum_{i=1}^{T} X_{i}-z>y \mid \sum_{i=1}^{T} X_{i}>z\right) .
\end{aligned}
$$

The third equality follows from the fact that

$$
\left[\left(T-n, S_{n+1}-x\right) \mid T>n, N(x)=n\right] \stackrel{\mathrm{D}}{=}\left(T^{n}, X_{n+1}\right),
$$

by the memoryless property of exponential random variables, and the fact that $T$ is independent of $S_{n}$ and $X_{n+1}$. Thus, $\sum_{i=1}^{T} X_{i}$ is IFR.

(b) This part can be proved similarly, except now $T^{n} \uparrow_{\text {st }} n$ and $X_{n} \uparrow$ st $n$, and the direction of the relevant inequalities must be changed.

Example 7.1. Let $X_{1} \stackrel{\mathrm{D}}{=} \operatorname{Exp}(\lambda), X_{i} \stackrel{\mathrm{D}}{=} \operatorname{Exp}(1), i \geq 2, T \stackrel{\mathrm{D}}{=} \operatorname{geometric}(p)$, and assume that these random variables are independent of one another. Since $[T-1 \mid T>1] \stackrel{\mathrm{D}}{=} \operatorname{geometric}(p)$, by the memoryless property of geometric random variables, it is easy to check that

$$
\left[\sum_{i=1}^{T-1} X_{1+i} \mid T>1\right] \stackrel{\mathrm{D}}{=} \operatorname{Exp}(p) .
$$

Therefore, we have

$$
\sum_{i=1}^{T} X_{i}=X_{1}+\sum_{i=1}^{T-1} X_{1+i} \mathbf{1}_{\{T>1\}} \stackrel{\mathrm{D}}{=} X_{1}+\hat{Y} \mathbf{1}_{\{T>1\}},
$$

where $\hat{Y}$ is an $\operatorname{Exp}(p)$ random variable independent of $X_{1}$ and $T$. If we assume that $p \neq \lambda$ then

$$
\begin{aligned}
\mathrm{P}\left(\sum_{i=1}^{T} X_{i}>x\right) & =\mathrm{P}\left(X_{1}+\hat{Y} \mathbf{1}_{\{T>1\}}>x\right) \\
& =\mathrm{P}\left(X_{1}>x\right)+\int_{0}^{x} \lambda \mathrm{e}^{-\lambda t} \mathrm{P}\left(X_{1}+Y>x, T>1 \mid X_{1}=t\right) \mathrm{d} t \\
& =1-\mathrm{e}^{-\lambda x}+\int_{0}^{x} \lambda \mathrm{e}^{-\lambda t}(1-p) \mathrm{e}^{-p(x-t)} \mathrm{d} t \\
& =\frac{\lambda(1-p)}{\lambda-p} \mathrm{e}^{-p x}+\frac{p(\lambda-1)}{\lambda-p} \mathrm{e}^{-\lambda x} .
\end{aligned}
$$


The failure rate function of $\sum_{i=1}^{T} X_{i}$ is therefore given by

$$
p+\frac{p(\lambda-1)}{p(\lambda-1) /(\lambda-p)+[\lambda(1-p) /(\lambda-p)] \mathrm{e}^{(\lambda-p) x}} .
$$

Since $\mathrm{e}^{(\lambda-p) x} /(\lambda-p) \uparrow x$ when $\lambda \neq p$, it follows from Theorem 7.1 that if $\lambda \geq 1$, then the failure rate function is nonincreasing, and that if $\lambda \leq 1$, then the failure rate function is nondecreasing.

Example 7.2. Let $X_{i}, i \geq 1$, be independent, identically $\operatorname{Exp}(\lambda)$-distributed random variables, and let $T$ be a geometric random variable independent of $X_{i}, i \geq 1$. Then

(a) $\max \left\{X_{1}, \ldots, X_{T}\right\}$ is IFR, and

(b) $T \max \left\{X_{1}, \ldots, X_{T}\right\}$ is DFR.

To see this, consider a parallel system with $n$ items whose lifetimes are $X_{1}, \ldots, X_{n}$, respectively. Then $\max \left\{X_{1}, \ldots, X_{n}\right\}$ is the time until the last item failure. Since the first failure time is an $\operatorname{Exp}(n \lambda)$ random variable and the time between the $i$ th failure and $(i+1)$ th failure, $i=1, \ldots, n-1$, is an $\operatorname{Exp}((n-i+1) \lambda)$ random variable, we have

$$
\left[\max \left\{X_{1}, \ldots, X_{T}\right\} \mid T=n\right] \stackrel{\mathrm{D}}{=} \sum_{i=1}^{n} Y_{i},
$$

where $Y_{i}$ is an $\operatorname{Exp}(i \lambda)$ random variable. Hence, $\max \left\{X_{1}, \ldots, X_{T}\right\} \stackrel{\mathrm{D}}{=} \sum_{i=1}^{T} Y_{i}$ and, by Theorem 7.1(a), we find that $\max \left\{X_{1}, \ldots, X_{T}\right\}$ is an IFR random variable.

Since $\left[T X_{i} \mid T=n\right] \stackrel{\mathrm{D}}{=} \operatorname{Exp}(\lambda / n)$, by using a similar argument we have

$$
\left[T \max \left\{X_{1}, \ldots, X_{T}\right\} \mid T=n\right] \stackrel{\mathrm{D}}{=} \sum_{i=1}^{n} Z_{i},
$$

where $Z_{i}$ is an $\operatorname{Exp}(\lambda / i)$ random variable. Hence, $T \max \left\{X_{1}, \ldots, X_{T}\right\} \stackrel{\mathrm{D}}{=} \sum_{i=1}^{T} Z_{i}$ and, by Theorem 7.1(b), we conclude that $T \max \left\{X_{1}, \ldots, X_{T}\right\}$ is a DFR random variable.

We now show that if the arrival rate of an inhomogeneous Poisson process is increasing, then the epoch time of a random IFR-distributed number of arrivals is itself IFR. Similarly, if the arrival rate is decreasing then the epoch time of a random DFR-distributed number of arrivals is DFR.

Theorem 7.2. Let $\lambda(t)$ be the intensity function of an inhomogeneous Poisson process $\{N(t)\}_{t \geq 0}$, and let $S_{n}$ be the nth event arrival time of this process. Assume that $T$ is a discrete random variable independent of $N(t), t \geq 0$.

(a) If $\lambda(t) \uparrow t$ and $T$ is IFR, then $S_{T}$ is IFR.

(b) If $\lambda(t) \downarrow t$ and $T$ is DFR, then $S_{T}$ is DFR.

The theorem can be proved using a technique similar to that used in the previous theorem, involving the independent increment property of Poisson processes. However, the following proof is more instructive. 
Proof of Theorem 7.2. (a) Again let $m(t)=\int_{0}^{t} \lambda(t) \mathrm{d} t$, meaning that $\left\{N\left(m^{-1}(t)\right)\right\}_{t \geq 0}$ is a homogeneous Poisson process with rate 1. Thus, if $\bar{S}_{n}$ is the $n$th arrival time of $\left\{N\left(m^{-1}(t)\right)\right\}_{t \geq 0}$, then $S_{n}=m^{-1}\left(\bar{S}_{n}\right)$ is the $n$th arrival time of $\{N(t)\}_{t \geq 0}$. Therefore,

$$
\begin{gathered}
\mathrm{P}\left(S_{T}>x\right)=\mathrm{P}\left(m^{-1}\left(\bar{S}_{T}\right)>x\right)=\mathrm{P}\left(\bar{S}_{T}>m(x)\right), \\
f_{S_{T}}(x)=f_{\bar{S}_{T}}(x) m^{\prime}(x), \\
r_{S_{T}}(x)=r_{\bar{S}_{T}}(m(x)) m^{\prime}(x)=r_{\bar{S}_{T}}(m(x)) \lambda(x) .
\end{gathered}
$$

Hence, to prove the theorem, it is enough to show that $r_{\bar{S}_{T}}(m(x)) \lambda(x) \uparrow x$. From Theorem 7.1 we know that $r_{S_{T}}(x) \uparrow x$ since $\bar{S}_{T}$ is the sum of $T \operatorname{Exp}(1)$ random variables and because $m(x) \uparrow x$. Thus, from the assumption that $\lambda(x) \uparrow x$, we find that $S_{T}$ is IFR.

(b) This part can be similarly proved using $\lambda(x) \downarrow x$ and the fact that $\bar{S}_{T}$ is DFR (see Theorem 7.1).

\section{Acknowledgements}

The careful attention and helpful suggestions of the referee are gratefully acknowledged. The research of SMS and ZZ was supported by the National Science Foundation grant ECS-0224779 with the University of California.

\section{References}

Barlow, R. E. And Marshall, A. W. (1964). Bounds for distributions with monotone hazard rate. Ann. Statist. 36, 1234-1274.

Barlow, R. E. And Marshall, A. W. (1965). Tables of bounds for distributions with monotone hazard rate. J. Amer. Statist. Assoc. 60, 872-890.

Barlow, R. E. And Proschan, F. (1975). Statistical Theory of Reliability and Life Testing. Holt, Rinehart and Winston, New York.

KiJima, M. (1989). Uniform monotonicity of Markov processes and its related properties. J. Operat. Res. Soc. Japan 32, 475-490.

KiJima, M. (1992). Further monotonicity properties of renewal processes. Adv. Appl. Prob. 25, 575-588.

Kochar, S. C. (1990). Some partial ordering results on record values. Commun. Statist. Theory Meth. 19, $299-306$.

Li, H. ANd ShaKed, M. (1997). Ageing first-passage times of Markov processes: a matrix approach. J. Appl. Prob. 34, $1-13$.

Pellerey, F., Shaked, M. And Zinn, J. (2000). Nonhomogeneous Poisson processes and logconcavity. Prob. Eng. Inf. Sci. 14, 353-373.

Ross, S. M. (2000). Introduction to Probability Models, 7th edn. Harcourt, Burlington, MA.

Shaked, M. and Shanthikumar, J. G. (1988). On the first-passage times of pure jump processes. J. Appl. Prob. 25, 501-509.

Shaked, M. And Shanthikumar, J. G. (1994). Stochastic Orders and Their Applications. Academic Press, Boston, MA.

Shanthikumar, J. G. (1988). DFR property of first-passage times and its preservation under geometric compounding. Ann. Prob. 16, 397-406. 